\title{
Analysis of Campus Card System Construction Based on Business
}

$$
\text { Huijie Zhu }{ }^{1, a} \text {, Chunbo Wei }{ }^{1, b} \text {, Guotao } X^{1, c} \text { and Lan Luan }{ }^{1, d}
$$

${ }^{1}$ Hunnan Road, Hunnan District, Shenyang City, Liaoning Province, Shenyang Jianzhu University

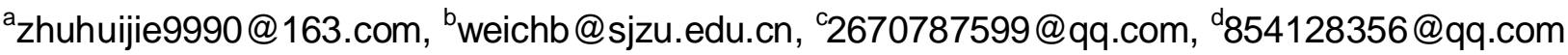

\section{Keywords: Business; Campus card; System; Authentication}

\begin{abstract}
Objective: This paper intends to build a business-based intelligent campus card information management system. Methods: Through the use of a variety of campus card system construction techniques, through detailed system analysis and research, a financial service center, a unified identity authentication center, and a data resource center have been designed. Results: Using the above technologies and methods, it provides theoretical support and technical assurance for the proposed business-based functional campus card system. Conclusion: Using the technique of this article, after careful analysis, it can be fully applied in the new digital application platform and satisfy the application of various systems in the school.
\end{abstract}

\section{Background}

The business-based campus card information management system is a collection of multiple payments and management-related systems created on the basis of the school's Internet environment ${ }^{[1]}$. Through the application on smart cards, the campus digital application platform is seamlessly integrated ${ }^{[2]}$. Through the unified identity authentication and data sharing center on the school's digital platform, all the various application systems in the school that require campus card applications are all integrated and integrated ${ }^{[3][4]}$. The campus card can truly replace all kinds of campus credentials, link various system applications into a multi-functional card application system, and achieve "holding a school card and a communication campus."

The business-based campus card information management system has been deeply involved in all aspects of the school's daily modern management and application, enabling schools to meet the world's standards ${ }^{[5]}$. It integrates the existing business and management independence and inconsistency to form an effective whole, avoid redundant construction, reduce costs, achieve business process reorganization, improve the overall management of the school, and become an indispensable daily essential for teachers and students ${ }^{[6]}$. It is based on the existing campus network, using digital high-tech management methods and its corresponding management equipment, the modernization of educational resource management, the autonomy of student learning and campus life, and the goal of sharing most of the campus' information resources ${ }^{[7]}$.

\section{Campus Card System Core Technology}

Campus Card System Appeared in domestic colleges and universities in the early 1990s. With only one card, multiple functions were realized, providing many conveniences for teachers and students. The first-generation campus card system has a relatively simple architecture. All application systems are directly connected to the RDBMS. The traditional data engine is used. The administrator can use the client software to directly access the core RDBMS. Since the first generation of systems used data engines such as ODBC, ADO, and BED to search database data, both databases and systems were on the same workstation ${ }^{[8]}$. There was no independent setup for RDBMS. The generation system also did not consider the docking problem with other systems, and therefore it could not be compatible with other applications, and its breadth was not enough. When the instantaneous big data interaction increases, the phenomenon of data congestion is easy to occur, which makes the system work energy efficiency is not high, and the user experience is poor. Based on this technology, the application server settings were later added to ease the system's access pressure on the RDBMS. The stability of the system was ensured through the application of the 
system server so that it would not be as easily congested as the first generation ${ }^{[9]}$.

Since entering 2008, Middleware technology has been gradually developed to construct a multi-layered structure and enhance the performance of this system. The middleware is to get the original data from the original signal acquisition and processing, and save it in the database, which is convenient for the background database to use the monitoring system to query and manage the data. Middleware technology is a key technology for constructing distributed applications and system integration. It is a distributed software that connects between system software such as operating systems and application software in the network environment. It is designed in the form of an API. A suite of software services enables servers, application services, and system-running controls in a networked environment to easily exchange information and exchange and assist at high speeds.

Analysis of Campus Card System Construction Based on Business. The business-based campus card information management system is an information system for all personnel in the entire campus. It has an important position in the information management of the campus. Therefore, in the design of the system must strictly implement the overall planning of information construction, adhere to norms, advanced, safe, reliable, open, practical, scientific, economic, and reasonable basis. When designing the system, it is necessary to consider from the following points, so that the system can meet the planning of the digitized construction of the entire campus information, and can effectively dock with other systems, and thus integrate into the entire digital campus. The design of the system follows the following principles

Specification and overall principle: The business-based campus card information management system not only integrates various application systems such as identity authentication, attendance, consumption, and library on the campus, but also meets the current requirements for digital campus construction. The interface with campus information portals and other management information systems, as well as third-party interface services based on web services technology, can be implemented to achieve all kinds of information on the campus's overall basic data.

Practical and flexible principle: It is the first element of a business-based campus card information management system that satisfies the cardholder's daily activities and the day-to-day management of the management side. Therefore, it is necessary to make full use of the safe and convenient self-service equipment and powerful digitization. The campus network creates an environment where teachers and students can self-service various businesses at any time and any place.

The principle of openness and scalability: To ensure the upward and downward compatibility of the system, the system needs to have open and extensible functions and access to other systems.

Network Architecture Design. The system construction of the hardware network should take the application as the core idea. In the design, the technical requirements of the campus education management, campus card system, and multimedia teaching should be fully taken into consideration. The technology should be advanced, and there must be sufficient conditions for future expansion. RS-485 This traditional control network is deployed in some low-speed and real-time control domains as the communication connection between these distributed control devices and data collection devices. For example, canteens and POS machines in the store transmit data through the RS485 protocol and the connected commercial gateways to achieve data collection and communication control of the terminal equipment. The on-board POS machine is connected to the business gateway through a wireless transmission protocol to achieve black and white list delivery and data collection. Self-service terminals, such as roll-in machines, self-service recharge machines, self-service cards, and administrator computers, transmit data through the TCP/IP protocol and the integrated front-end machine. Through the division of VLANs in each network area, they will be mixed. The logical separation between terminals and campus networks in the campus network. Through the transmission of Layer 3 switches and core routers, it is connected to the campus card system private network to ensure the security of the campus card system private network. The campus card system private network and the bank private network carry out secure network data transmission through the IOS8583 protocol. 


\section{Analysis of Campus Card System Components Based on Business}

Financial Service Center Design. The financial service center is the core and most basic service of the business-based campus card information management system. It mainly deals with the management of transaction services in the entire process of card-based tokens, that is, all data related to financial transactions are processed through financial service centers. Responsible for querying and analyzing financial data. There is a service platform, a reporting system and an integrated business system. The service platform is a platform that integrates multiple services to achieve unified display and unified management, providing cardholders with a convenient one-stop service. The use of $\mathrm{B} / \mathrm{S}$ architecture to design and use, to ensure that users can access login through web pages at any time and anywhere, to provide users with personal account information maintenance, payment, inquiry, transfer and other services. Unify the various types of self-service terminals on campus, expand new terminal applications such as mobile phones, give full play to terminal service promotion capabilities, and promote integrated applications such as consumer payment, information inquiry, booking, and media promotion.

The report system uses the $\mathrm{B} / \mathrm{S}$ architecture design to create and analyze various financial reports provided by the financial service center, and then provides report queries and merchant queries. Its main functional modules include Web-http services, report services, and menu resource licensing services. The integrated business system is the core business operation platform for the entire financial service center and is based on the C/S structure design. The cardholder's center, merchant center, inquiry center, report center, maintenance center, and security center compose a comprehensive service management for cardholders.

Unified Identity Management Center Design. The unified identity management center is a business-based campus card information management system management module. All subsystems are authorized and user identity authentication is performed through unified identity management, and all system operations are monitored uniformly. Confirming the same identity on all kinds of system applications in the school network is to perform identification on the chip card at the terminal: calling the cardholder's information data, the legitimacy of the chip card, and then performing the resolution on the credential bank library to compare the information data. There is a unified identity authentication center, monitoring system and management platform. The unified identity authentication center provides unified identity management and authentication services to all subsystems, and establishes a unified identity information center of the entire school according to the unified standards and rules based on the identity data existing in the campus business systems. The adoption of a unified identity standard facilitates the comprehensive utilization of identity-related business data by users. Based on unified identity data and a unified technology interface, it provides identity authentication services to various business systems on campus. Externally provide single sign-on (SSO) authentication service, identity information authentication service (Web Service), through the well-defined external interface, to provide authorized third-party applications with identity information data, the acquired data range and content must be able to define . Considering that the management user of the unified identity authentication center is fixed and the information security is highly demanded, the $\mathrm{C} / \mathrm{S}$ structure design with high security is used.

The monitoring system monitors and records all equipment and system operations in the entire system in real time. Record and alarm the abnormal fault point. Monitors whether the site settings are monitored at the end, end geographical area, end geographic coordinates, abnormal configuration, and other functions. The end (that is, each service or end) sends a status package to the monitoring center, and monitors the configuration of the central credential monitoring site to process and archive the monitored site. Topological maps, electronic maps, etc., show the status information at the end. It is also possible to write flow and operation logs from various subsystems to the database and monitor the websites for direct query and display.

In order to better monitor the status of various devices, the monitoring system adopts the B/S structure to design, which can better improve the reusability of some of its functional modules, and greatly facilitate the management of management personnel and reduce the management cost. The 
management platform adopts the $\mathrm{B} / \mathrm{S}$ structure design to integrate all business systems under the business-based campus card information management system to realize unified login, unified display, and unified management of all business systems in the business-based campus card information management system. Continuity of business process; Through a unified identity authentication, to achieve a single sign-on system, can be seamlessly switched in each registered subsystem, without having to log in again, effectively improving the system's operating efficiency; will be integrated System business resources are presented to management personnel in the form of application services, which satisfy the individualized customization and business requirements of management personnel.

Data Resource Center Design. The data resource center is the integration of all business data to the application system center, the establishment of data warehouse, the use of data integration technology, and the flow data and unified identity management center of the financial service center based on the business-based campus card information management system. The data dictionary and other data are loaded into the data warehouse for use by subsystems. In order to ensure data security and meet the needs of convenient use, the Data Resource Center integrates the unified authorization management center's unified authorization and single sign-on functions, establishes a complete data query interface, and provides third-party systems with a standardized standardized historical data. . Third-party systems can make in-depth data mining analysis after acquiring data. Data mining is to hide those data from the data. It has not been understood and there are valid data extracted. Through the establishment of a computer algorithm program, this information is actively investigated in the database to discover its discipline or model.

\section{Summary}

This article describes in detail the overall design of the service-based smart card campus card information management system, first introduced the design principles, then described the overall structure and overall architecture design of the system, and then described in detail the design of the system operating environment, and finally proposed The design plan of the business-based campus card information management system is elaborated in detail from three aspects: the design of the financial service center, the design of the unified identity management center, and the data resource center.

\section{References}

[1] M.C.TSAI,K.H.LAI.A study of the institutional forces influencing the adoption intention of RFID by suppliers. Information \& Management ,2012

[2] H.SUPIC. Project management maturity of selected organizations in Croatia. Proceedings of $8^{\text {th }}$ International Conference on Telecommunications . 2015: 239-245

[3] I.A.ALMERHAG.Key Length as a Qos routing metric. Woodward M E. Sixth Informatics Workshop.2005: 233-245

[4]Suhaiza Zailani, Liu Wen Sze,Yudi Fernando.Determinants of RFID Adoption in Supply Chain among Manufacturing Companies in China: A Discriminant Analysis. Journal of Technology Management \& Innovation. 2017

[5] Hong Qu, Jiuyuan Huo.2012.Design and Implementation of Digital Campus Project in University.Advanced Information Technology in Education, AISC 126:89 94

[6] Qian Wang, Nai Jia Liu, and Zhi Rui Cheng. 2012. The Application Research of Data Exchange Technology in Digital Campus. ISc IDE 2011, LNCS 7202, 607-613

[7] Xianmin Wei.2011.Research of College Information Integration and Sharing System. International Conference on Advances in Education and Management ISAEBD 2011.Part IV, CCIS 211: 615 619

[8] Duan Z.-M, Wang R.-L,Sun M.-Q.2008.Research and Implementation of the Digital Campus Resource Integration Based on Packaged Campus Cards. Computer Engineering\&Science, $30(1), 8 \sim 11$ 
[9] Chirathamjaree C, Mukviboonchai S.2002.The Mediated Integration Architecture for Heterogeneous Data Integration. IEEE TENCON,2: 89 92 